\title{
Multi-lineages of Shiikuwasha (Citrus depressa Hayata) evaluated by using whole chloroplast genome sequences and its bio-diversity in Okinawa, Japan
}

\author{
Ryuji Ishikawa*1), Nathan Badenoch ${ }^{2)}$, Kunimasa Miyagi ${ }^{3)}$, Kaname Medoruma ${ }^{4)}$, Toshiki Osada ${ }^{5}$ \\ and Masayuki Onishi ${ }^{6}$ \\ 1) Faculty of Agriculture and Life Science, Hirosaki University, Hirosaki, Aomori 036-8561, Japan \\ 2) Center for Southeast Asian Studies, Kyoto University, Kyoto, Kyoto 606-8501, Japan \\ 3) Association of Shishigaki Network, Naha, Okinawa 902-0071, Japan \\ 4) Okinawa prefectural Chūbu Agricultural Development Center, Department of Agriculture, Forestry and Fisheries, Okinawa Prefec- \\ ture, Nago, Okinawa 904-2155, Japan \\ 5) Research Institute of Humanity and Nature, Kyoto, Kyoto 603-8047, Japan \\ 6) Research Center for Knowledge Science in Cultural Heritage, Doshisha University, Kyoto, Kyoto 610-0394, Japan
}

Shiikuwasha (Citrus depressa Hayata) is distributed from the South-west of the Japanese archipelago to Taiwan. In this study, re-sequencing against the orange (C. sinensis (L.) Osbeck) chloroplast genome was applied to one superior landrace of Shiikuwasha cultivated in Oku ward, Okinawa, Japan. The chloroplast genome of the landrace was estimated to comprise $160,118 \mathrm{bp}$, including 48 indels and 71 nucleotide substitutions against the reference genome. The presumptive chloroplast indels were confirmed by subsequent experiments, and these identified multiple maternal lineages among other landraces. Some of the orange SSR markers were available for genotyping of other superior landraces and were able to distinguish among them. These molecular markers were then applied for evaluation of genetic diversity among wild and cultivated Shiikuwasha accessions. Except for Oku ward, the cultivated populations were found to have lost their genetic diversity in comparison with wild populations. Groves in Oku ward maintained, or showed even higher genetic diversity than wild accessions in the surrounding areas by the force of villagers.

Key Words: Citrus depressa Hayata, Shiikuwasha, re-sequencing, maternal lineage, selection.

\section{Introduction}

Wild relatives within the genus Citrus are distributed from East Asia to Oceania. Many horticultural species are considered to have their origins in these areas, and fruit production is very rich worldwide. For example, more than $68 \mathrm{Mt}$ of oranges and $131 \mathrm{Mt}$ of other citrus fruits were produced globally in 2012 (FAO Stats, http://faostat3.fao.org). Taxonomical relationships among Citrus species are, however, enormously complicated; on the basis of independent taxonomical considerations, Swingle (1943) identified 10 species, whereas Tanaka (1954) identified 147, including horticultural species.

Vegetative propagation has contributed to the spread of horticultural Citrus species. For example, cultivation of the sweet orange (C. sinensis (L.) Osbeck) has been widespread,

Communicated by K. Kato

Received November 18, 2015. Accepted April 19, 2016.

First Published Online in J-STAGE on July 9, 2016.

*Corresponding author (e-mail: ishikawa@hirosaki-u.ac.jp) but details about its origin have not been clear. Based on molecular data (Xu et al. 2013), it is presumed that the species was created through a process of domestication involving hybridization between the pomelo (C. maxima (Burm.) Merr.), synonymously $C$. grandis L.) and the mandarin orange (C. reticulata Marcow.), followed by additional hybridization between the mandarin and the F1 hybrid. The grapefruit probably originated from cross hybridization between the orange and the pomelo. In addition to crosshybridization breeding, bud mutation has complicated the origins of Citrus species. For example, detection of bud mutations might be involved in the origin of Citurs varieties. For example, bud mutation and mutation breeding using mutagens have enabled breeders to establish further varieties of grapefruit (Moore 2001). Most of the Citrus species currently found in Japan were introduced from abroad and they were subsequently improved by bud-mutation breeding and cross-hybridization as in the case of orange and grapefruit.

Okinawa is one of the regions where Citrus species have been reported in abundance, and two wild species are 
present there (Tanaka 1948, 1957). One of these native species, C. depressa Hayata (a common name; Shiikuwasha), is distributed in the south-west part of the Japanese archipelago and in mountainous areas of Taiwan. Tachibana (C. tachibana (Makino) Tanaka) is another wild species distributed in Japan, but is not as popular as other Citrus fruits at the commercial level. As Shiikuwasha carries more heterozygous loci than Tachibana, it appears to be a hybrid between Tachibana and an unknown species (Yamamoto et al. 1998). However, Shiikuwasha shows a variety of morphologic features in terms of fruit size, shape, and appearance, suggesting that it may not have originated from a single cross, but rather through independent domestication from a wild population involving multiple events. In fact, for a very long time it has been tradition for local farmers to introduce wild plants into their groves. Language studies also support the idea of multiple domestication because there are various local names for specific accessions. Some are designated "Kugani", "Kachi", or "Tanibuta", meaning gold, thick peel, or large seeds (Kinjo 2007, Onishi and Miyagi 2016). Despite an abundance of local names, the accessions corresponding to each are not known precisely. Therefore, there is still a chance of finding valuable resources such as a seedless accession (Medoruma et al. 2011).

In order to ascertain the origin of Shiikuwasha and its degree of genetic diversity, intensive molecular studies are required, but no effective tool for studying Shiikuwsha existed until the development of Citrus genome studies. The orange is one Citrus species that is dominant worldwide, and genome studies have revealed the full chloroplast genome sequence and a partial nuclear genome sequence (Bausher et al. 2006, Xu et al. 2013). In the present study, using the chloroplast genome of the orange as a referencegenome, we subjected Shiikuwasha to next generation sequencing (NGS). Nuclear SSR markers used to construct an orange linkage map, were also applied to Shiikuwasha, although it was not possible to amplify most of them. These molecular tools demonstrated that polymorphism does exist among Shiikuwasha landraces. Accordingly, landraces and wild accessions were collected in Okinawa in order to evaluate their degree of diversity. We found that recent cultivation had resulted in a dramatic reduction of diversity, and also that genetic variability still exists in the northern parts of Okinawa island.

\section{Materials and Methods}

\section{Plant materials}

In order to screen for genetic polymorphism, domesticated and wild accessions of Shiikuwasha were collected from three areas of Okinawa prefecture, Japan, during 2013-2014: Ogimi village, Nago city, and Kunigami village (Table 1, Fig. 1). All materials were donated by local villagers and farmers, and taken from groves that were owned by them. Leaves and fruit from wild individuals that were not endangered species were collected from public areas. Ogimi village is the most well-known area for Shiikuwasha production, and has 29 domesticated accessions. Seventy-six wild accessions were collected as a wild population at Nekumachiji Mountain in Ogimi village. Katsuyama ward in Nago city is

Table 1. Shiikuwasha materials collected in Okinawa, Japan

\begin{tabular}{llc}
\hline \hline Site & \multicolumn{1}{c}{ Sites } & No. of accessions \\
\hline Oogimi & Domesticated & 29 \\
& Wild & 76 \\
Nago & Domesticated & 21 \\
& Wild & 44 \\
Kunigami & Domesticated & 190 \\
& Wild & 75 \\
\hline & Total & 435 \\
\hline
\end{tabular}

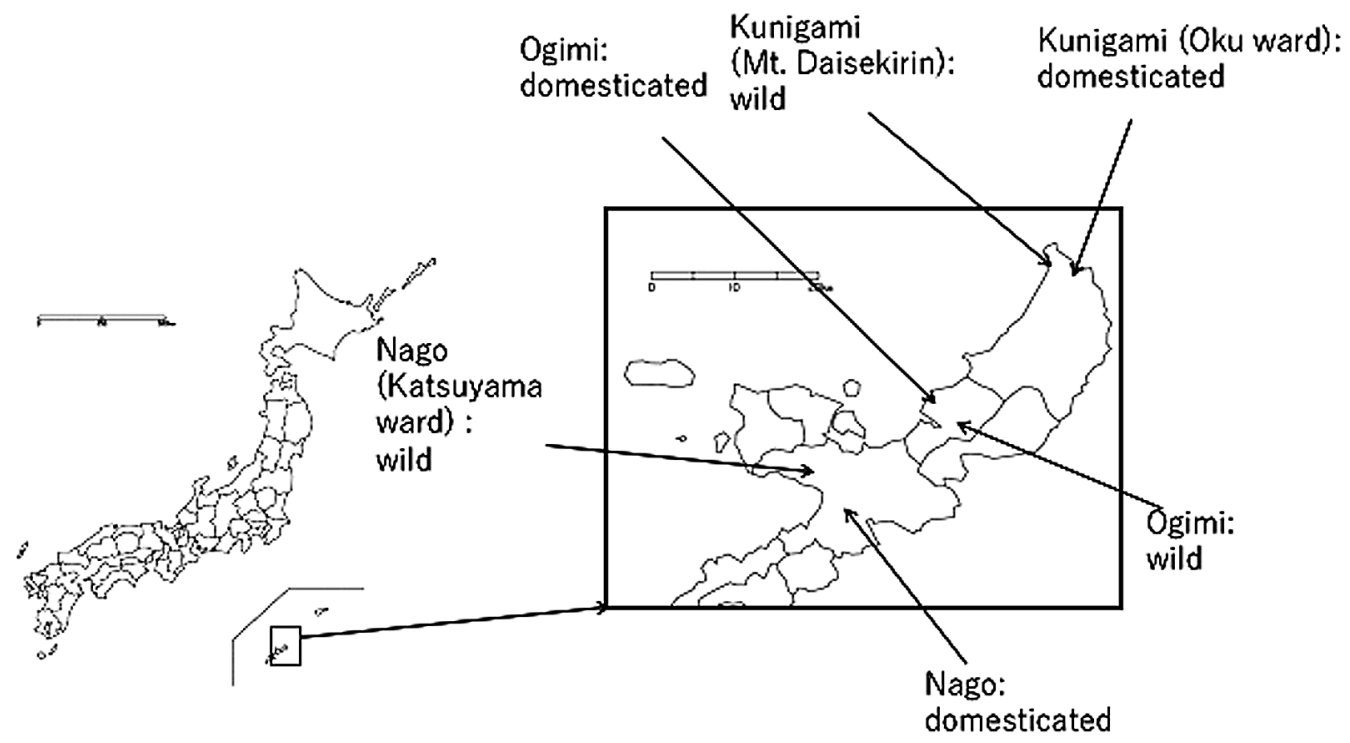

Fig. 1. Collection sites in Okinawa, Japan. 
Table 2. Chloroplast and nuclear SSR markers and chloroplast INDELs (cpINDEL) to evaluate polymorphism in chloroplast and nuclear genome

\begin{tabular}{|c|c|c|c|c|c|c|}
\hline \multirow{2}{*}{ Type } & \multirow{2}{*}{ Markers } & \multirow{2}{*}{$\begin{array}{c}\text { Abbrevi- } \\
\text { ation }\end{array}$} & \multirow{2}{*}{ Forward } & \multirow{2}{*}{ Reverse } & \multicolumn{2}{|c|}{ Genetic diversity* } \\
\hline & & & & & Oku ward & Daisekirin-zan \\
\hline \multirow{8}{*}{$\begin{array}{l}\text { Cytoplasmic } \\
\text { marker }\end{array}$} & MM 2-cpINDEL1 & & TAATTGGAACCCTAGGGCTC & ACTGCGACTGATCCTGAAAGG & 0.018 & 0.000 \\
\hline & MM 2-cpINDEL2 & & GCTTGAAAAAGCCCGTCTTTAC & AATGATAGAAATGGAATTGCTG & 0.018 & 0.000 \\
\hline & MM 2-cpINDEL3 & & GGATTCCATTTCCGAGTACC & GTATCAATGGTCACTCTCTGG & 0.489 & 0.000 \\
\hline & MM 2-cpINDEL4 & & GATCAATTGAGATATCCGGAAACC & GGTTAGTCCTTGAACCGAGTG & 0.053 & 0.000 \\
\hline & MM 2-cpINDEL5 & & GATATGAGATCTACAAATCTCC & CCCTTGGGTCTAATACAACAAC & 0.557 & 0.000 \\
\hline & MM 2-cpINDEL6 & & GTGGCTTCAACCTCAGCGAAACC & GCTAAATCGCAGTCCAGTATTTC & 0.018 & 0.000 \\
\hline & MM 2-cpINDEL7 & & CGAATTTTTATTCAACCCTATAG & CTATACGGTTCGAACTATCTAC & 0.413 & 0.080 \\
\hline & MM 2-cpINDEL8 & & CTTTTTTCTAAAGGTAGTTAGTG & GAAGTACGTTTTTTTGGAACTG & 0.053 & 0.000 \\
\hline \multirow[t]{8}{*}{ Nuclear } & Ma3_98 & 98 & GATCACCACAAGCAGCACAC & TCTCAAGAGCCCAGTTCGAT & 0.132 & 0.077 \\
\hline & Ma3_142 & 142 & TTTCTTCGTCAACCCCAAAG & TAGAAGAAGGACCCCGAACC & 0.266 & 0.186 \\
\hline & Ma3_168 & 168 & GATTCTTCTTTCGGCTGCTG & GCTCGACAGGTTGTTGGTTT & 0.533 & 0.756 \\
\hline & $168 \mathrm{rev}$ & & GTTGGTTTTGGTGAGTTTGG & GCGGCAGCATCAGAAGAATG & - & - \\
\hline & Ma3_200 & 200 & ATGTCGACGTTGACCAATGA & TTTCGCTTCTCAGGTGGACT & 0.375 & 0.484 \\
\hline & Ma2_582 & 582 & GACATGTCACACAAGCAGGC & GCAAAATACAGTTGAAAATGCG & 0.000 & 0.497 \\
\hline & $\mathrm{Ma} 2$ & 1710 & TGGAACATTGAAGTGGGTGA & ACTTGAGATTAGGGCCGGTT & 0.649 & 0.630 \\
\hline & 1710̄ev & & & ACACTAACCTCAGCCTCATAAG & - & - \\
\hline
\end{tabular}

* cpINDEL markers were genotyped with 110 Oku acessions and 24 Daisekiin mountain wild accessions.

168rev and 1710rev were modified to fit to score by sequencing gels with shiikuwasha samples.

a well-known center for manufacture of Shiikuwasha juice. Here, 21 domesticated accessions were collected. Wild accessions were collected from Katsu Mountain in Nago city, where wild Shiikuwasha was reported to be abundant nearly 60 years ago (Tanaka 1957). At Oku ward in Kunigami village, 190 domesticated accessions were collected because various phenotypes were observed in groves there. Wild accessions were also collected at Daisekirin Mountain in Kunigami village. Leaves from these collected accessions were used for extraction of DNA using the general urea method. In order to evaluate variation in fruit characteristics, three fruits per tree were harvested from 41 trees in Oku ward and 17 trees in Ogimi village in October, 2014. Fruit skin thickness was measured with a digital caliper, DCN100 (Mitsutoyo Co., Japan). Acidity (\%) was measured with a PAL-ACID1 device (Atago Co., Ltd., Japan). Percentage sugar content (BRIX) was measured with a PAL1 device (Atago Co., Ltd.). Seven superior landraces (Nakamotoseedless, Ishi-kunibu, Izumi-kugani, Katsuyama-kugani, Asahikawa-Shiiuwasha, Fusubuta, and Ogimi-kugani) were employed as control plant materials stocked at Okinawa Agricultural Research Center (Nago branch). MM2, which is a late-ripening landrace present in Oku ward, was used for detailed comparison of genotypes with a superior landrace.

\section{Molecular markers}

SSR markers developed for the orange nuclear genome were applied to Shiikuwasha (Xu et al. 2013). Only seven markers among 29 nuclear SSRs examined were able to genotype; the others could not be amplified (Supplemental Table 1). One of the seven markers was excluded later because it was not stably amplified (Ma3 5). These markers were then used to measure molecular diversity (Table 2). Chloroplast markers developed using other Citrus species were then applied to Shiikuwasha (Cheng et al. 2005), and four of them were useful for measurement of Shiikuwasha diversity. These markers were amplified using PCR cycles of $94^{\circ} \mathrm{C}$ for $3 \mathrm{~min}, 30$ rounds of $95^{\circ} \mathrm{C}$ for $10 \mathrm{~s}, 55^{\circ} \mathrm{C}$ for $30 \mathrm{~s}$, and $72^{\circ} \mathrm{C}$ for $30 \mathrm{~s}$, and finally $72^{\circ} \mathrm{C}$ for $5 \mathrm{~min}$ with Thermopol (NEB Ltd., Japan). The amplified DNA fragments were elecrophoresed on $6 \%$ denaturing polyacrylamide gel at $1500 \mathrm{~V}$ for $2 \mathrm{~h}$ in $0.5 \mathrm{X}$ TBE. The gels were then stained with silver nitrate.

Evaluation of the full chloroplast sequence using next generation sequencing (NGS)

The superior landrace, MM2, sampled in Oku ward (GPS:N2651305/E12815160) under a permission of the owner was used for DNA extraction with a Dneasy Plant Mini Kit (QIAGEN Co., Japan). After libraries had been prepared with a 350-bp insert-TrueSeq Nano DNA LT Sample Prek kit, 100-bp pair-end reads were obtained by HiSeq 1000 (Illumina Inc., Japan), and this yielded 52,666,382 reads corresponding to $5,318 \mathrm{Mb}$ in total size. The High Quality Base $(30<\mathrm{A})$ content was $92.49 \%$. Re-sequencing analysis against the $C$. sinensis chloroplast genome (NC_008334) was performed using CLC Genomics Workbench (ver 7.0). Reads were covered for 178 times on average against the orange chloroplast genome. Indels of more than $4 \mathrm{bp}$ against the reference genome were screened. Primers were designed to amplify a fragment of about $200 \mathrm{bp}$ covering each indel deduced in the orange genome (Table 2). Then, Shiikuwasha accessions were genotyped with the standard SSR protocol as described above.

\section{Results}

\section{Variations in morphological and physiological traits}

Fruits were collected from 58 trees to evaluate variations in morphological and physiological traits (Fig. 2). Three fruits were measured per single individual for acidity and Brix. In general, acidity gradually decreased until Jaunary. 
A.

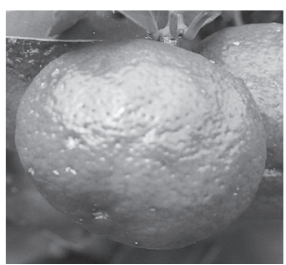

B.

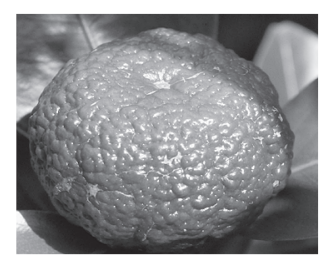

C.

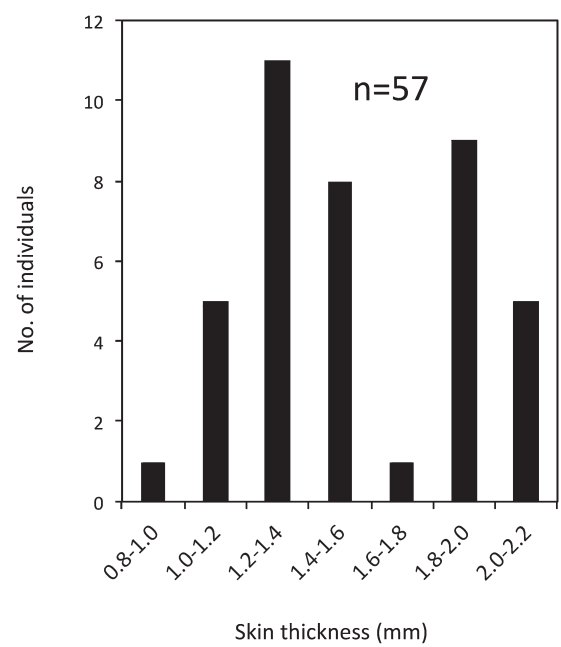

D.

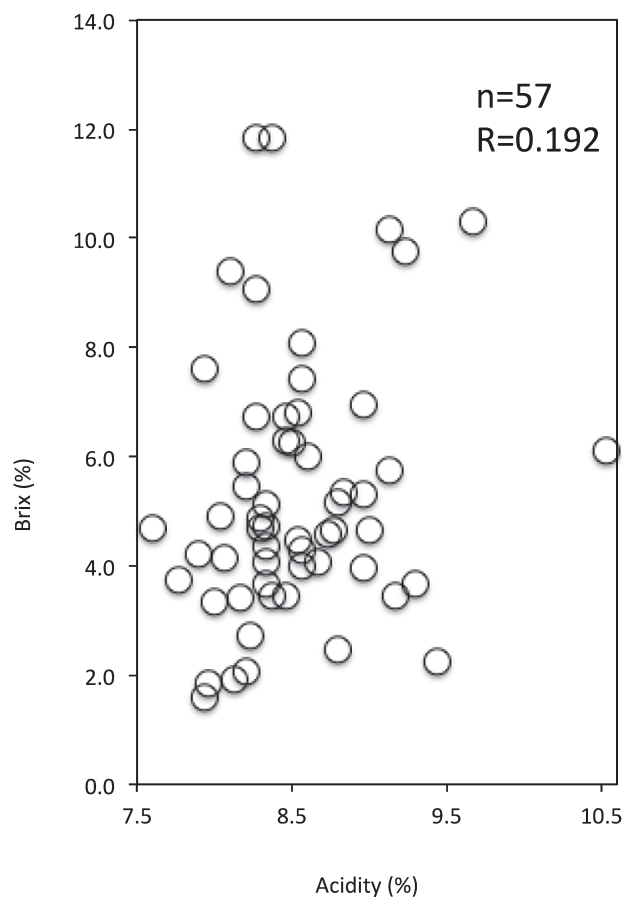

Fig. 2. A. Fruit with smooth surface called as Kugani type generated abundant fruit juice. B. Fruit with rough surface called as "Kachi" type generated fragrance. C. Fruit skin thickness represented discontinuous distribution. D. Fruit phenotypes, Acidity and Brix (\%) with 57 accessions measured by three fruits of single individual trees. There was no significant correlation between the traits. D. Collection sites wild and domesticated accessions were obtained.

However, immature fruits are harvested starting in October. Compared to other Citrus species, consumers prefer the sour taste of Shiikuwasha. The thickness of the fruit skins showed discontinuous variation with two modes of 1.2-1.4 and $1.8-2.0 \mathrm{~mm}$. These ranged from 0.9 to $2.1 \mathrm{~mm}$ in Oku ward and from 0.8 to $1.6 \mathrm{~mm}$ in Ogimi village, and thus variation in fruit skin was wider in Oku ward. The acidity scores ranged from $7.6 \%$ to $10.5 \%$, and the Brix scores from $1.6 \%$ to $11.9 \%$. The distributions of both scores were continuous. The Oku population showed a wider range of acidity. The minimum acidity score was $7.6 \%$ in the Oku population, which was $0.57 \%$ lower than that in the Ogimi population. The Brix score in the Oku population was higher than that in the Ogimi population.

\section{Chloroplast markers of the orange genome}

In order to develop molecular markers for $C$. depressa Hayata, re-sequencing of a superior accession from Oku ward in Kunigami village was performed. The average depth was x178 against the reference chloroplast genome, which is 160,129 bp in size (NC_008334). Indels and SNP variants were screened in comparison with the reference genome. Twenty-one deletions, 26 insertions, one multinucleotide variant, and 71 SNPs were detected (Supplemental Tables 2, 3). The flanking sequences of the corresponding regions tended to carry single nucleotide repeats or duplications of short sequences. In total, the chloroplast genome of $C$. depressa Hayata was estimated to be $160,118 \mathrm{bp}$ in size. The complete chloroplast genome sequence has been registered in the DDBJ as Submission ID: $5714 a 8902113 a 5 b 85901557 c$. Based on the genome sequence, more than $4 \mathrm{bp}$ of chloroplast insertion/deletion (MM2-cpINDEL)s were developed as INDEL markers and confirmed experimentally (Fig. 3, Supplemental Table 3). Three markers-MM2-cpINDEL3, MM2-cpINDEL4, and MM2-cpINDEL5 - carried more than three alleles when wild and cultivated accessions were genotyped (data not sown). A deletion of CTCTTTTTT inside MM2-cpINDEL3 was flanked with 10 thymine repeats, whereas polymorphism in MM2-cpINDEL4 was based on single adenine repeats. The MM2-cpINDEL5 was an insertion of AATTTG at a tandem duplication of the AATTTG motif. These nucleotide structures resulted in the generation of multiple alleles.

Among the cpINDELs, eight MM2-cpINDELs were further analyzed. For six out of eight MM2-cpINDELs, alternative alleles were observed among landraces (Table 3). The indel markers and categorized landraces formed two groups. Group 1 comprised Nakamoto-seedless and Ishikunibu, whereas Group 2 comprised Izumi-Kugani, Fusubuta, Katsuyama-Kugani, Asahikawa-Shiikuwasha, and Ogimi-Kugani. MM2 carried genotypes different from 
A.

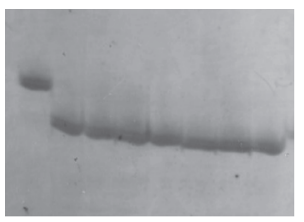

E.

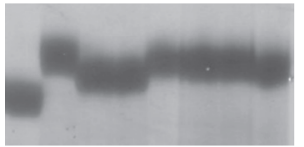

B.

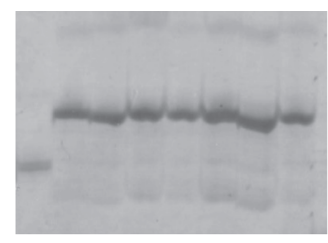

F.

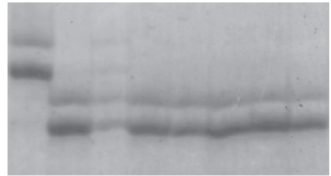

C.

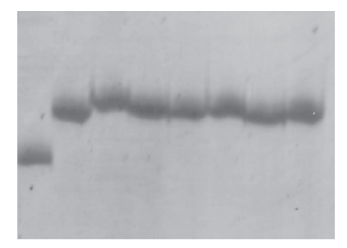

D.

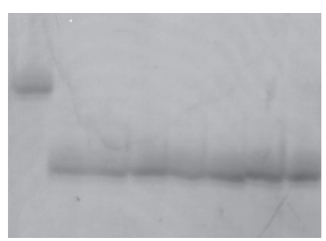

G.

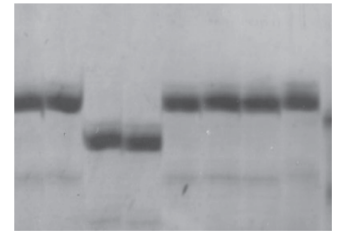

H.

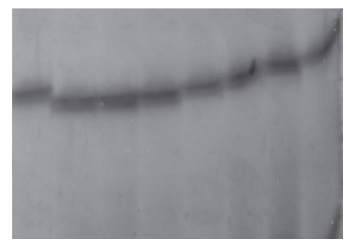

Fig. 3. Maternal traceability accessed with eight chloroplast INDELs developed from a whole genome sequence data obtained from MM2 (lane 1). From lane 1 to lane 8, MM2, genotypes of Oogimi kugani, Nakamoto seedless, Ishi kunib, Izumi kugani, Asahikawa shiikuwasha, and Fusubuta were shown.

Table 3. Discrimination of single landraces with eight chloroplast INDELs developed with NGS data and six nuclear SSR markers

\begin{tabular}{|c|c|c|c|c|c|c|c|c|c|c|c|c|c|c|}
\hline \multirow{2}{*}{ Cultivars } & \multicolumn{8}{|c|}{ MM2-cpINDEL } & \multicolumn{6}{|c|}{ Nuclear markers } \\
\hline & $\mathrm{C} 1$ & $\mathrm{C} 2$ & $\mathrm{C} 3$ & $\mathrm{C} 4 *$ & $\mathrm{C} 5$ & C6 & $\mathrm{C} 7$ & $\mathrm{C} 8$ & 98 & 142 & 168 & 200 & 582 & 1710 \\
\hline MM2 & 2 & 1 & 1 & 3 & 1 & 2 & 2 & 2 & $2 / 3$ & $1 / 3$ & $2 / 2$ & $1 / 2$ & $3 / 3$ & $1 / 2$ \\
\hline Nakamoto-seedless & 1 & 2 & 3 & 1 & 2 & 1 & 1 & 1 & $2 / 2$ & $2 / 2$ & $2 / 2$ & $1 / 2$ & $3 / 3$ & $1 / 3$ \\
\hline Ishi-kunibu & 1 & 2 & 3 & 1 & 2 & 1 & 1 & 1 & $2 / 2$ & $2 / 2$ & $2 / 2$ & $1 / 1$ & $3 / 3$ & $1 / 2$ \\
\hline Izumi-Kugani & 1 & 2 & 2 & 1 & 3 & 1 & 2 & 1 & $2 / 2$ & $2 / 2$ & $2 / 3$ & $1 / 1$ & $3 / 3$ & $1 / 4$ \\
\hline Katsuyama-Kugani & 1 & 2 & 2 & 1 & 3 & 1 & 2 & 1 & $2 / 2$ & $2 / 2$ & $2 / 4$ & $1 / 1$ & $3 / 3$ & $1 / 4$ \\
\hline Asahikawa-Shiiuwasha & 1 & 2 & 2 & 1 & 3 & 1 & 2 & 1 & $2 / 2$ & $2 / 2$ & $2 / 4$ & $1 / 1$ & $3 / 3$ & $1 / 4$ \\
\hline Fusubuta & 1 & 2 & 2 & 1 & 3 & 1 & 2 & 1 & $2 / 2$ & $2 / 2$ & $2 / 2$ & $1 / 2$ & $3 / 3$ & $1 / 4$ \\
\hline Ogimi-Kugani & 1 & 2 & 2 & 1 & 3 & 1 & 2 & 1 & $2 / 2$ & $2 / 2$ & $2 / 4$ & $1 / 1$ & $3 / 3$ & $1 / 4$ \\
\hline
\end{tabular}

* Multiple allele, 2 was observed in wild accessions (data not shown).

all the others. Although $110 \mathrm{Oku}$ accessions were genotyped with eight MM2-cpINDELs, only MM2 carried alternative alleles at three loci and a few accessions shared the same alleles with MM2 (Table 2). Three markers-MM2cpINDEL3, 5, and 7-showed relatively high genetic diversity, at $0.489,0.557$, and 0.413 , respectively. The genetic diversity of other markers ranged from 0.018 to 0.053 . In all cases, MM2 carried unique genotypes in comparison to most of the others, except for MM2-cpINDEL7.

\section{Development of nuclear markers}

SSR markers in C. sinensis (L.) Osbeck were selected randomly and applied to $C$. depressa Hayata. Twenty-one out of 26 SSR markers were successfully amplified, and of these, four markers were monomorphic among the examined accessions. The remaining 17 markers showed polymorphisms, of which six were applied in order to determine the genotypes of superior landraces. Apart from two landraces - Fusubuta and Ogimi-Kugani- the rest were genetically different and distinguishable from each other. Genotypes were then compared between landraces and wild accessions, both having been collected in Oku ward. Al- though the Oku landraces shared a single genotype at Ma2_582, all other markers showed polymorphism in both populations. These landraces showed relatively high diversity, with averaged $\mathrm{He}$ scores ranging from 0.089 to 0.588 .

\section{Genetic diversity in Okinawa}

Shiikuwasha farming is widespread in the northern half of Okinawa island. Leaf samples of cultivated Shiikuwasha accessions were collected from two villages (Ogimi and Kunigami) and one city (Nago). Wild accessions were detected in forests near these sites.

In total, 345 accessions collected from Ogimi village, Nago city, and Kunigami village were genotyped with the three MM2-cpINDELs. Domesticated accessions in Kunigami village originated only in Oku ward. Fifteen genotype combinations were recognized as plastid types (Table 4). The genotype of MM2 accessions was 1-1-2 (MM2-cpINDEL3, 5 , and 7), which was not shared with other domesticated or wild individuals. Among domesticated populations, the Ogimi and Nago populations were composed of only one or two genotypic combinations. Although wild populations were composed of a greater number of genotypic combinations, 
Table 4. Chloroplast genotypes measured with three chloroplast INDELs developed with NGS data of MM2 accessions, compared among domesticated and wild accessions in three regions, Ogimi village, Nago city, and Kunigami village

\begin{tabular}{|c|c|c|c|c|c|c|c|c|c|}
\hline \multirow{3}{*}{$\begin{array}{l}\text { Maternal } \\
\text { lineage }\end{array}$} & \multirow{2}{*}{\multicolumn{3}{|c|}{ MM2-cpINDEL }} & \multicolumn{6}{|c|}{ No. of accessions } \\
\hline & & & & \multicolumn{2}{|c|}{ Ogimi } & \multicolumn{2}{|c|}{ Nago } & \multicolumn{2}{|c|}{ Kunigami } \\
\hline & cpINDEL3 & cpINDEL5 & cpINDEL7 & Dom. & Wild & Dom. & Wild & Dom. & Wild \\
\hline Type1 & 1 & 1 & 1 & 0 & 0 & 0 & 0 & 1 & 0 \\
\hline Type2 & 1 & 1 & 2 & 0 & 0 & 0 & 0 & 1 & 0 \\
\hline Type3 & 1 & 2 & 1 & 0 & 0 & 0 & 0 & 3 & 0 \\
\hline Type4 & 1 & 2 & 2 & 0 & 64 & 0 & 0 & 8 & 0 \\
\hline Type 5 & 1 & 3 & 2 & 0 & 8 & 0 & 0 & 53 & 0 \\
\hline Type6 & 2 & 1 & 1 & 0 & 0 & 0 & 0 & 5 & 0 \\
\hline Type 7 & 2 & 2 & 1 & 0 & 1 & 7 & 1 & 24 & 17 \\
\hline Type8 & 2 & 2 & 2 & 29 & 0 & 13 & 18 & 15 & 41 \\
\hline Type9 & 2 & 3 & 1 & 0 & 0 & 0 & 2 & 0 & 0 \\
\hline Type10 & 2 & 3 & 2 & 0 & 0 & 0 & 7 & 4 & 4 \\
\hline Type11 & 3 & 1 & 1 & 0 & 0 & 0 & 0 & 0 & 1 \\
\hline Type12 & 3 & 2 & 1 & 0 & 0 & 0 & 8 & 0 & 4 \\
\hline Type13 & 3 & 2 & 2 & 0 & 0 & 0 & 3 & 0 & 0 \\
\hline Type14 & 3 & 3 & 1 & 0 & 0 & 0 & 1 & 0 & 0 \\
\hline Type15 & 3 & 3 & 2 & 0 & 0 & 0 & 2 & 0 & 0 \\
\hline Total & & & & 29 & 73 & 20 & 42 & 114 & 67 \\
\hline
\end{tabular}
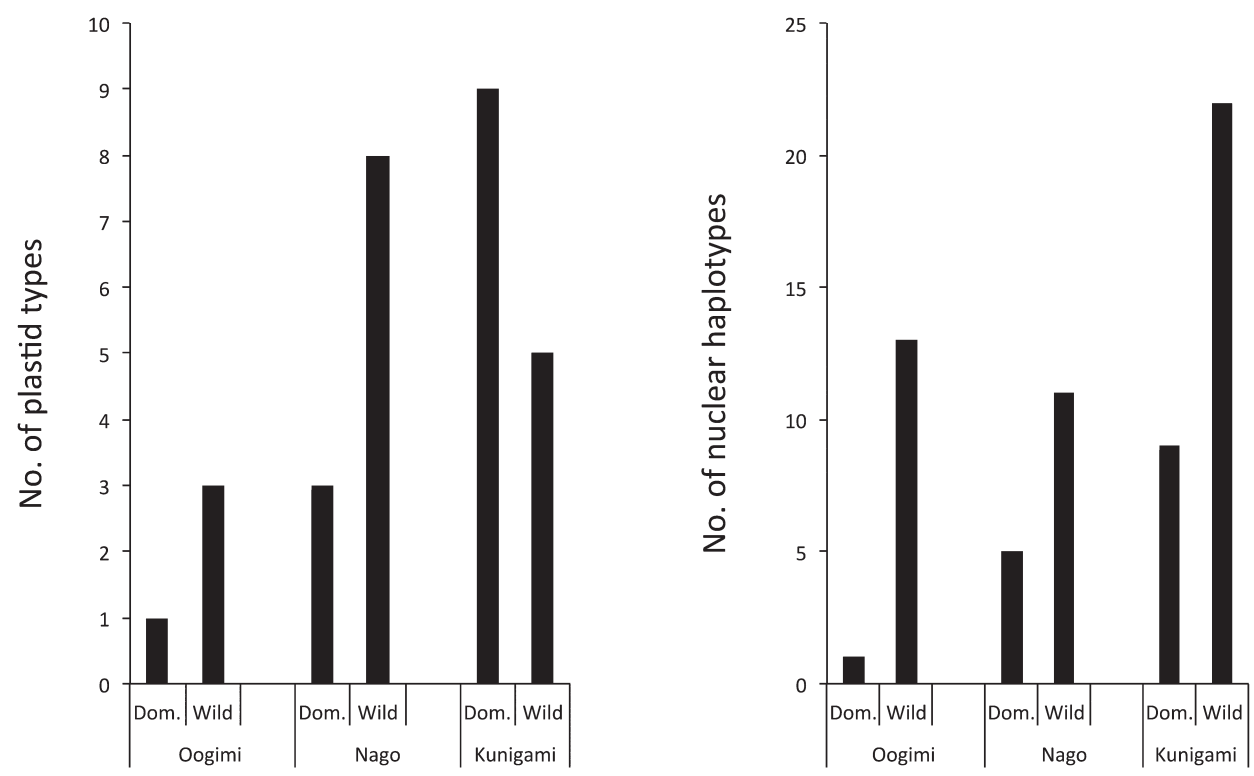

Fig. 4. Number of haplotypes detected among wild and domesticated populations at three sites. Haplotypes were determined with two loci, Ma3_168 and Ma3_200.

there were only three or eight combinations in the Ogimi and Nago populations, respectively (Fig. 4, Supplemental Table 4). The most varied combinations were identified in a population collected in Oku ward. Nine genotypic combinations including one MM2 were observed. In contrast, five genotypic combinations were detected in a wild population in Kunigami village.

Two nuclear markers-Ma3_168 and Ma3_200-were genotyped with wild and domesticated accessions. Multiple alleles at the Ma3 168 locus did not give good resolution for genotyping. A new primer pair was therefore designed based on the sequence corresponding to the Ma3 168 locus, and this primer pair yielded good resolution. The genotypes of the two markers were defined as haplotypes. The highest number of haplotypes (nine) was found in Kunigami village (Table 5). Only one haplotype was found in Ogimi village, compared to 13 haplotypes in wild populations near the farms.

\section{Apomixis}

In the past, local farmers established their groves with stocks from wild trees or by transplantation of young seedlings from the surrounding habitats. This led to recognition of several superior accessions, which were conserved as key landraces as shown in Table 3. For this reason, trees in modern groves did not show variation. For Shiikuwasha, there had been no data about apomixis, which is one of the mechanisms by which variation can be reduced. Therefore, 
Table 5. Nuclear haplotypes combined with two nuclear SSR markers, Ma3_168 (168) and Ma3_200 (200) among domesticated and wild accessions in three regions, Ogimi village, Nago city, and Kunigami village

\begin{tabular}{|c|c|c|c|c|c|c|c|c|}
\hline \multirow{3}{*}{ Haplotype } & \multirow{2}{*}{\multicolumn{2}{|c|}{ Haplotype }} & \multicolumn{6}{|c|}{ No. of accessions } \\
\hline & & & \multicolumn{2}{|c|}{ Ogimi } & \multicolumn{2}{|c|}{ Nago } & \multicolumn{2}{|c|}{ Kunigami } \\
\hline & 168 & 200 & Dom. & Wild & Dom. & Wild & Dom. & Wild \\
\hline Hap1 & $1 / 1$ & $1 / 1$ & 0 & 1 & 0 & 0 & 1 & 0 \\
\hline Hap2 & $1 / 1$ & $1 / 2$ & 0 & 0 & 0 & 2 & 0 & 7 \\
\hline Hap3 & $1 / 1$ & $1 / 2$ & 0 & 0 & 0 & 1 & 0 & 0 \\
\hline Hap4 & $1 / 2$ & $1 / 1$ & 0 & 3 & 0 & 0 & 0 & 1 \\
\hline Hap5 & $1 / 2$ & $1 / 2$ & 0 & 3 & 0 & 1 & 0 & 3 \\
\hline Hap6 & $1 / 2$ & $1 / 2$ & 0 & 1 & 0 & 0 & 0 & 2 \\
\hline Hap7 & $1 / 3$ & $1 / 2$ & 0 & 1 & 0 & 0 & 0 & 2 \\
\hline Hap8 & $1 / 3$ & $2 / 2$ & 0 & 0 & 0 & 0 & 0 & 1 \\
\hline Hap9 & $1 / 4$ & $1 / 1$ & 0 & 1 & 0 & 0 & 0 & 0 \\
\hline Hap 10 & $1 / 4$ & $1 / 2$ & 0 & 1 & 0 & 1 & 0 & 1 \\
\hline Hap11 & $1 / 5$ & $1 / 1$ & 0 & 1 & 0 & 0 & 0 & 0 \\
\hline Hap12 & $2 / 2$ & $1 / 1$ & 0 & 0 & 1 & 3 & 10 & 4 \\
\hline Hap13 & $2 / 2$ & $1 / 2$ & 0 & 0 & 7 & 27 & 19 & 4 \\
\hline Hap14 & $2 / 2$ & $2 / 2$ & 0 & 1 & 0 & 0 & 0 & 0 \\
\hline Hap 15 & $2 / 3$ & $1 / 1$ & 0 & 0 & 1 & 0 & 4 & 2 \\
\hline Hap16 & $2 / 3$ & $1 / 2$ & 0 & 0 & 2 & 1 & 6 & 2 \\
\hline Hap17 & $2 / 4$ & $1 / 1$ & 29 & 47 & 9 & 2 & 57 & 11 \\
\hline Hap18 & $2 / 4$ & $1 / 2$ & 0 & 8 & 0 & 2 & 10 & 10 \\
\hline Hap19 & $2 / 4$ & $2 / 2$ & 0 & 0 & 0 & 0 & 6 & 4 \\
\hline Hap20 & $2 / 5$ & $1 / 1$ & 0 & 0 & 0 & 0 & 1 & 0 \\
\hline Hap21 & $3 / 3$ & $1 / 1$ & 0 & 0 & 0 & 0 & 0 & 1 \\
\hline Hap22 & $3 / 3$ & $1 / 2$ & 0 & 0 & 0 & 1 & 0 & 4 \\
\hline Hap23 & $3 / 3$ & $2 / 2$ & 0 & 0 & 0 & 0 & 0 & 1 \\
\hline Hap24 & $3 / 4$ & $1 / 1$ & 0 & 0 & 0 & 0 & 0 & 1 \\
\hline Hap25 & $3 / 4$ & $1 / 2$ & 0 & 0 & 0 & 0 & 0 & 3 \\
\hline Hap26 & $3 / 4$ & $2 / 2$ & 0 & 0 & 0 & 1 & 0 & 1 \\
\hline Hap27 & $4 / 4$ & $1 / 1$ & 0 & 2 & 0 & 0 & 0 & 1 \\
\hline Hap28 & $4 / 4$ & $1 / 2$ & 0 & 0 & 0 & 0 & 0 & 1 \\
\hline Hap29 & $4 / 4$ & $2 / 2$ & 0 & 1 & 0 & 0 & 0 & 0 \\
\hline Total & & & 29 & 71 & 20 & 42 & 114 & 67 \\
\hline
\end{tabular}

progenies propagated through seeds were genotyped and compared with their parental trees. Seeds from one superior tree (MM2) and one wild accession (Oku) next to the old junior high school in Oku ward were collected. Seeds from each tree were germinated and allowed to grow from separated poly-embryos inside the seeds (Fig. 4). Young leaves from single seedlings were then taken for extraction of DNA and genotyping with six SSRs separately. One of 16 young seedlings (6.3\%) from the MM2 tree and two of eight seedlings $(25 \%)$ differed in genotype from the mother tree. For example, a codomionant marker, Ma3 168 showed a non-parental allele associated with crossing between the Oku tree and other tree(s) (Fig. 5). The difference in the percentage among seedlings suggested that outcrossing would have been influenced by the surrounding conditions. The tree from the Oku ward, was a wild one that stood alone, whereas MM2 stood at the top of a hilly area in a grove. Possible pollen donors could not be determined because of a lack of genetic information about the surrounding trees at present. A high rate of apomixis is one possible reason for the low diversiy in Ogimi village, where extreme proliferation through seeds of particular superior accessions has occurred. In contrast, it is also suggested that careful selection to screen the progeny derived from cross-hybridization might allow breeders to improve Shiikuwasha through crossing within the species.

\section{Discussion}

\section{Cultivation history and local selection processes}

Shiikuwasha is the only Citrus species in Japan that is commercially cultivated and co-exists in a wild form in forests. Therefore it may have high tolerance to biotic or abiotic stress. No other varieties of popular cultivated fruits, such as Oto, Tsukurubu, or Kabuchi, are found under wild conditions in Okinawa. Shiikuwasha has long been consumed in the form of vinegar, and has been used as a substitute bleaching solution. To this day, it is quite common to find trees in individual home gardens and yards. The historical record of the Shiikuwasha is documented in the Omorozoushi, an Okinawan text that was edited in the mid 16th to
A.

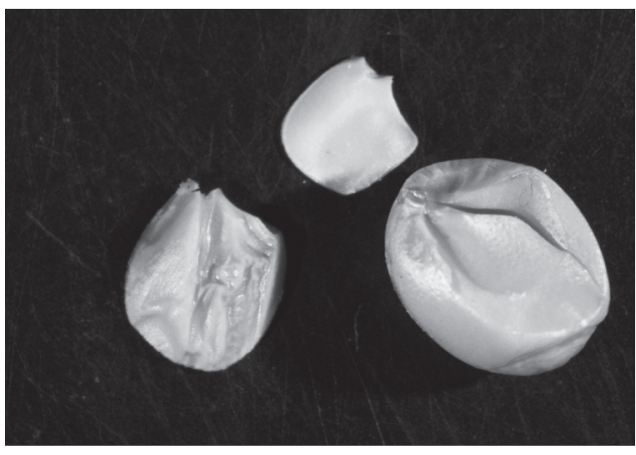

B.

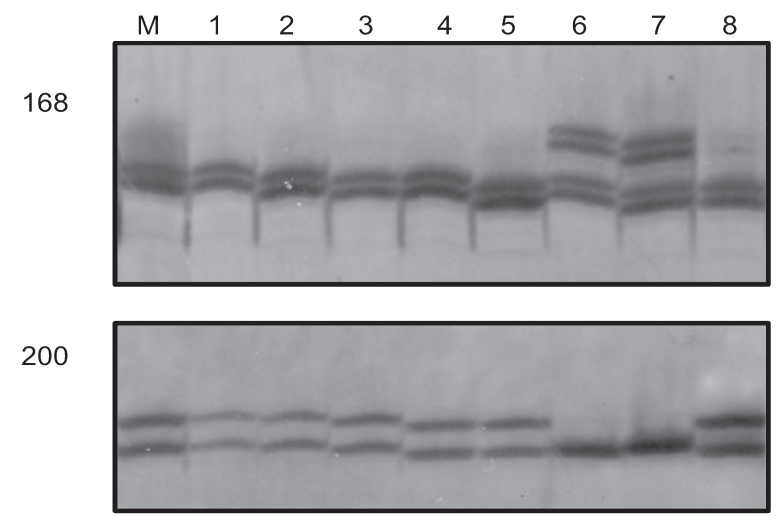

Fig. 5. Polyembryos in a single seeds and polymorphisms detected in progenies of single trees. A. polyembryos detected in a single seed. B. Polymorphisms found by using Ma3 168 and Ma3 200 markers in progeny seedling of the Oku tree. Genotypes of Ma3 168 and Ma3 200 suggested that No. 6 and No. 7 seedlings outcrossed with other plants. 
17th centuries (Kinjo 2007). However, commercial cultivation began only about 50 years ago. Since then, landraces have been cultivated in restricted areas of central and northern Okinawa island. Consumption of Shiikuwasha increased gradually in the 1980s when the juice industry began to take off. Food science research subsequently identified high levels of vitamin $\mathrm{C}$ and other health benefits, futher boosting the popularity of the Shiikuwasha (Kinjo 2007). This intensive period of commerical development necessitated the amplification of vegetative clones for expansion of cultivation in areas of central Okinawa, such as Katsuyama ward and Ogimi village. Although market forces have tended to homogenize the industry, a significant variation in cultivated Shiikuwasha is still evident. One example of this variation is fruit skin thickness, which shows a bimodal distribution. These traits may be partly affected by environmental conditions on a broader scale, but genetic factors are generally important as well. Experienced farmers have selected superior individuals at their farms. Against the interplay of these factors, breeders have succeeded in selecting superior accessions and maintained them at Okinawa Prefectural Institute of Agriculture.

Molecular markers make it possible to evaluate differences among genetic resources. However, there are few molecular markers in Shiikuwasha that yield high resolution. At present, the orange is one Citrus species for which a large amount of genetic information is available. In order to develop molecular tools for examination of Shiikuwasha, we obtained re-sequencing data against the orange chloroplast genome, and this helped us to develop chloroplast indel markers for distinguishing between landraces. This approach also revealed multiple maternal lineages in the cpINDELs, suggesting that the origin of Shiikuwasha is complex. Multiple domestication, or a single domestication event followed by a long period to allow accumulation of DNA mutations, are the most likely scenarios. As shown in Tables 4, 5, wild populations demonstrated wider variation than cultivated ones. It implies that the domestication was happened in Okinawa, Japan.

Katsuyama ward and Ogimi village showed markedly fewer different maternal lineages in comparison with the multiple lineages in Oku Ward. Nuclear genotypes also showed the same trend. The number of haplotypes was highest in a wild population collected from Daisekirin mountain in Kunigami village. In contrast, the number of maternal lineages was higher in Oku ward than in any of the wild populations. This diversity is a direct result of the introduction of other accessions from different areas into Oku ward, in addition to the ease with which clones from wild trees can be developed. Oku is located in the northern-most area of the main island of Okinawa. Local people keep their own Shiikuwasha trees in home gardens and yards, while utilizing wild plants that occur nearby. When compared with Ogimi village and Katsuyama Ward, Oku farmers allowed, and in fact encouraged, marked variation in their groves, ranging from sour to sweet fruit, and early to late flowering individuals. These have been maintained locally through the conscious efforts of the farmers.

In attempting to clarify the extent of diversity in Shiikuwasha, it should be noted that, historically, Oku ward has remained relatively isolated. The local language, rituals and ecological knowledge that supports the people's traditional livelihood of the local population have all been preserved until very recently (Onishi and Miyagi 2016). In response to the cultural and economic pressures that accompany increased integration into contemporary Okinawan society, the village has developed several strategies for conserving their culture. A village museum has been established to document and transmit the history of the village. Community leaders explained to us how their culture is intimately related to the surrounding environment and their livelihood activities; they keep a wide range of Shiikuwasha fruits in their groves. We hypothesize that their culture, which stresses the importance of maintaining their local language and rituals, may have contributed to the maintenance of plant diversity, including Shiikuwasha. The villagers of Oku demonstrate a locally based, yet deep understanding of biocultural diversity that underpins the genetic diversity that has been observed in our study. This kind of diversity is important for screening of valuable genetic stock and making improvements to it.

\section{NGS data and traceability}

The fruits of accession MM2 are of superb quality. In addition, the significantly late maturity is unique, in comparison to other Shiikuwasha accessions. On the basis of its indels, this accession was determined as plastid Type 14 . These indels were based on DNA polymorphism between the orange chloroplast genome and the re-sequenced genome based on reads of the MM2 accession. NGS is able to provide genetic tools such as indels markers for characterization of non-model plants or unique landraces such as the MM2 tree. For certification of food security or organic cultivation, genetic diversity and chloroplast genome information is cost-effective, and recent biochemical data suggest that Shiikuwasha fruit may be beneficial to human health in addition to contributing to the local economy. Molecular tools for identifying individual accessions may help to improve the agronomy of the Shiikuwasha. Although the production of this fruit is deeply influenced by environmental conditions such as soil, nutrients, water content, and probably other unknown factors, genetic identification followed by cultivation experiments may provide important insights into the necessary conditions for obtaining good fruit.

\section{Center of diversity}

The significant diversity found in local residential areas tended to be secondary centers of diversity, where various genetic components have accumulated in particular crops (Vavilov 1926). Secondary centers of biodiversity in residential areas are a potential source of further domestication for new candidate crops (Anderson1954, Nakao 1966). 
Transplanting of wild Shiikuwasha trees and detection of superior individuals are frequently performed by villagers in Oku ward. As Shiikuwasha has recently been established as a commercial fruit, wild populations and populations in secondary centers such as Oku may be a good breeding resource for a pure-selection methodology which could apply to existing varieties.

The question still remains as to why the villagers in $\mathrm{Oku}$ have maintained this high level of diversity, not only in terms of local culture but also Shiikuwasha production. The answer may lie the spirit of the villagers themeselves, who place high value on diversity in general. Domestication activities have resulted in the introduction of genetically unique individuals such as the MM2 accession, which carries a unique plastid type with superior quality. These accessions, the relatively low rate of apomixis, and molecular markers will allow breeders to develop linkage maps and recognize linkage disequilibria on the basis of phenotypic data.

\section{Acknowledgements}

We thank local coordinators and famers in Oku Ward for their help in collecting valuable materials from local farmers.

\section{Literature Cited}

Anderson, E. (1954) Plants, man and life. A. Melrose Ltd., London Bausher, M.G., N.D. Singh, S.-B.Lee, R.K. Jansen and H.Daniell (2006) The complete chloroplast genome sequence of Citrus sinensis (L.) Osbeck var 'Ridge Pineapple': organization and phylogenetic relationships to other angiosperms. BMS Plant Biol. 6: 21.

Cheng,Y., M.C.D.Vicente, H.Meng, W.Guo, N.Tao and X.Deng
(2005) A set of primers for analyzing chloroplast DNA diversity in Citrus and relatd genera. Tree Physiol. 25: 661-672.

Kinjo,H. (2007) About shiikuwasha in South-east archaeopelago. Studia Citologica 17: 137-148.

Medoruma,K., A.Higa, H.Kinjyo, H.Zukeyama, Y.Awaguni, T. Miyagi, M.Arasaki, H. Inoue, S. Onda, S. Kawano et al. (2011) Characteristics of seedless Citrus depressa, Nakamoto seedless. Bull. Okinawa Pref. Agric. Res. Center 5: 5-10.

Moore,G.A. (2001) Oranges and lemons: clues to the taxonomy of Citrus from molecular markers. Trends Genet. 17: 536-540.

Nakao, S. (1966) Origin of cultivated plants and Agriculture. Iwanami, Tokyo, Japan.

Onishi,M. and K. Miyagi (2016) Wisdom in Shiikuwasha-Oku/ Yanbaru, Circulation among dialect, local community, life. Kyoto University Press, Kyoto, Japan, p. 529.

Swingle, W.T. (1943) The botany of Citrus and its wild relatives of the orange subfamily. In: Webber,H.J. and L.D. Batchelor (eds.) The Citrus Industry. Vol. I, University of California Press, USA, pp. $129-474$.

Tanaka,T. (1954) Species problem in citrus: a critical study of wild and cultivated units of citrus, based upon field studies in their native homes (Revisio Aurantiacearum IX), Japanese Society for the Promotion of Science, pp. 141-152.

Tanaka,T. (1957) Citrus in Ryukyu. Department of Economics, Ryukyu government, pp. 31-37.

Tanaka, Y. (1948) An iconograph of Japanese Citrus fruits-A monographic study of species and varieties of Citrus fruits grown in Japan Vol. 2. Yokendo, Tokyo, Japan, pp. 481-488.

Vavilov, N.I. (1926) Studies on the origin of cultivated plants. Inst. Bot. Appl. Amelior. Plants, Leningrad, pp. 248.

Xu, Q., L.-L.Chen, X.Ruan, D.Chen, A.Zhu, C.Chen, D. Bertland, W.-B. Jiao, B.-H.Hao, M.P. Lyon et al. (2013) The draft genome of sweet orange (Citrus sinensis). Nat. Genet. 45: 59-68.

Yamamoto, M., Y.Matsuo, T.Kuniga, R.Matsumoto and Y.Yamada (1998) Isozyme and RAPD analyses of Shiikuwashas (Citurs depressa Hayata). Bull. Natl. Inst. Fruit Tree Sci. 30/31: 39-51. 